\title{
Length Distributions in Loop Soups
}

\author{
Adam Nahum and J. T. Chalker \\ Theoretical Physics, Oxford University, 1 Keble Road, Oxford OX1 3NP, United Kingdom \\ P. Serna, M. Ortuño and A. M. Somoza \\ Departamento de Física - CIOyN, Universidad de Murcia, Murcia 30.071, Spain
}

(Dated: August 1, 2013)

\begin{abstract}
Statistical lattice ensembles of loops in three or more dimensions typically have phases in which the longest loops fill a finite fraction of the system. In such phases it is natural to ask about the distribution of loop lengths. We show how to calculate moments of these distributions using $C P^{n-1}$ or $R P^{n-1}$ and $O(n) \sigma$ models together with replica techniques. The resulting joint length distribution for macroscopic loops is Poisson-Dirichlet with a parameter $\theta$ fixed by the loop fugacity and by symmetries of the ensemble. We also discuss features of the length distribution for shorter loops, and use numerical simulations to test and illustrate our conclusions.
\end{abstract}

Introduction. Statistical models for ensembles of loops arise in many areas of physics and the probability distribution of loop lengths offers an important way of characterising them. One expects that, although properties of the shortest loops are model-specific, universal features should emerge on longer scales. In this paper we present results for loop length distributions, obtained using simple field-theoretic methods and tested via Monte Carlo simulation. Considering systems in which some loops are extended, we find the joint length distribution of macroscopic loops via a calculation of moments that is exact (though not rigorous) in the relevant limit, showing that it is Poisson-Dirichlet (PD), a distribution [1] with many applications in statistics and probability theory.

Loop ensembles are generated in a wide variety of problems from statistical physics. They are central objects in polymer theory [2] and series expansions [3. They appear in vertex models 4, in the Coulomb phase of frustrated magnets [5] 7], and in studies of resonating valence bond wavefunctions [8, 9. An important class consists of vortex lines in three-dimensional random fields. These are of interest in settings ranging from cosmology [10] to liquid crystals [11] and optics [12, and lattice formulations 13 have long been used for precise numerical simulations. More broadly, there are parallels 14 between problems involving the length distributions of cycles in random maps, the replica theory of spin glasses, and Levy flights.

Loops and cycles also appear naturally for many-body quantum systems viewed in imaginary time, when particle trajectories make up strands that close either via creation and annihilation processes or under time-periodic boundary conditions [15. A key application is to quantum Monte Carlo simulations, in which properties of a system are computed by sampling the loop ensemble [16].

Loop and cycle models have likewise attracted considerable attention in the mathematical physics and statistics literature, both as probabilistic representations of quantum spin systems [17] and as statistical problems in their own right. In particular, for a mean field-like prob- lem of cycles on the complete graph, the distribution of cycle lengths has been shown to be PD [18. Recently Ueltschi and collaborators [19 have made the striking conjecture, with support from simulations [20, that the same form also applies for long loops in three-dimensional systems. In this paper we provide a field-theoretic derivation of this result.

As we discuss in the following, two features are sufficient to fix the universal behaviour in these ensembles. One is whether or not the loops are directed. The second is the fugacity associated with loops in the ensemble.

Our starting point is a class of models for loops on lattices that we have defined in detail elsewhere [21 25]. These models may be formulated for either directed or undirected loops. They have configurations $\mathcal{C}$ consisting of completely-packed coverings of the lattice by loops, in which each link is part of one and only one loop. Coverings carry a statistical weight that is a product of two factors. One depends on the local arrangement of loops at nodes and a control parameter $p$. The other varies with the number of loops $|\mathcal{C}|$ in the configuration as $n^{|\mathcal{C}|}$, giving loops a fugacity that can be generated by summing over $n$ possible 'colourings' of each loop. The models support phases of two types: one in which all loops are finite, and another in which some loops are extended. Our concern here is only with behaviour in extended phases, reached via suitable choices for $p$ and the lattice.

We have found [22, 25] that a continuum description of these lattice models is provided by $\sigma$ models with the target space $C P^{n-1}$ or $R P^{n-1}$ depending on whether loops are directed or undirected. Moreover, $R P^{n-1}$ and $O(n)$ models are equivalent for our purposes, since the $Z_{2}$ gauge symmetry that distinguish them plays no role in the extended phases we are concerned with. Here we show how the $\sigma$ model formulation allows a calculation of all moments of the length distribution for extended loops. In the extended phase the $\sigma$ model has long range order: the computations reduce to a finite-dimensional averages over orientations of the order parameter, which can be evaluated exactly. 
Calculations. An outline of these calculations is as follows. We discuss initially directed loops, indicating changes needed for the undirected case later. The $\sigma$ model field $Q$ is an $n \times n$ traceless Hermitian matrix that can be parameterised in terms of an $n$-component complex vector $\mathbf{z}$ of length $\mathbf{z}^{\dagger} \mathbf{z}=n$ as

$$
Q^{\alpha \beta}=z^{\alpha} \bar{z}^{\beta}-\delta^{\alpha \beta} .
$$

Configurational averages in dimension $d$ are computed using the weight $e^{-S}$ with

$$
S=\frac{1}{2 g} \int \mathrm{d}^{d} x \operatorname{tr}(\nabla Q)^{2} .
$$

The $n$ values of the indices $\alpha$ and $\beta$ arise from possible loop colours and elements of $Q$, used as observables, select loop configurations from the ensemble. For instance, $Q^{\alpha \beta}(x)$ for $\alpha \neq \beta$ is a two-leg operator that, at the point $x$, absorbs a strand of colour $\alpha$ and emits one of colour $\beta$. The average $\left\langle Q^{12}\left(x_{1}\right) \ldots Q^{m 1}\left(x_{m}\right)\right\rangle$ is hence proportional to the weight in the ensemble for a loop to pass in order through the points $x_{1} \ldots x_{m}$, with its colour taking the values 1 to $m$ on successive segments, instead of being a free variable.

We use these ingredients to calculate moments of the loop length distribution [26. Let $l_{1}, l_{2} \ldots$ be the lengths of loops in a configuration, in decreasing order. Then

$$
\begin{aligned}
\int \mathrm{d}^{d} x_{1} \ldots \mathrm{d}^{d} x_{m}\left\langle Q^{12}\left(x_{1}\right) \ldots Q^{m 1}\left(x_{m}\right)\right\rangle & \\
= & \frac{A^{m}}{n(m-1) !}\left\langle\sum_{i} l_{i}^{m}\right\rangle,
\end{aligned}
$$

where $A$ is the nonuniversal normalisation associated with the operator $Q$, the factor of $(m-1)$ ! arises because the points $x_{1} \ldots x_{m}$ appear in a prescribed order around the selected loop, and the factor of $n$ is because there is no sum on the colour of this loop. In the ordered phase, which occurs at small $g$ for $d>2$, the dominant contribution to the above $m$-point function comes from the spatially constant part of $Q^{\alpha \beta}(x)$, which is reduced in magnitude by fluctuations compared to (1). We therefore set

$$
Q^{\alpha \beta}(x) \simeq B\left(z^{\alpha} \bar{z}^{\beta}-\delta^{\alpha \beta}\right),
$$

where $B$ is determined by the strength of long-range order. The correlator is given by averaging $\mathbf{z}$ over the sphere $\mathbf{z}^{\dagger} \mathbf{z}=n$, representing possible directions of broken symmetry. This yields

$$
\begin{aligned}
\left\langle Q^{12}\left(x_{1}\right) \ldots Q^{m 1}\left(x_{m}\right)\right\rangle & \simeq B^{m}\left\langle\left|z^{1}\right|^{2} \ldots\left|z^{m}\right|^{2}\right\rangle \\
& =B^{m} \frac{n^{m} \Gamma(n)}{\Gamma(n+m)} .
\end{aligned}
$$

Combining expressions, taking the system volume (the total length of all loops) to be $\mathcal{L}$, and writing $n B / A=f$, we have

$$
\mathcal{L}^{-m}\left\langle\sum_{i} l_{i}^{m}\right\rangle=f^{m} \frac{n \Gamma(n) \Gamma(m)}{\Gamma(n+m)}
$$

Since we retained only the spatially uniform part of $Q^{\alpha \beta}(x)$ in Eq. (4), this result applies in the thermodynamic limit $\mathcal{L} \rightarrow \infty$.

To have full information on the joint probability distribution of loop lengths we must calculate general moments of the form

$$
\left\langle\sum_{i_{1}, \ldots, i_{q}}^{\prime} l_{i_{1}}^{m_{1}} \ldots l_{i_{q}}^{m_{q}}\right\rangle .
$$

Here the prime on the sum indicates that $i_{1}, \ldots, i_{q}$ are distinct loops. This average is easily related to the integral of the $\left(\sum_{k=1}^{q} m_{k}\right)$-point correlation function

$$
\left\langle\Gamma^{(1)}\left(x_{1}^{(1)}, \ldots, x_{m_{1}}^{(1)}\right) \ldots \Gamma^{(q)}\left(x_{1}^{(q)}, \ldots, x_{m_{q}}^{(q)}\right)\right\rangle,
$$

in which $\Gamma^{(k)}$ forces the coordinates $x_{1}^{(k)}, \ldots x_{m_{k}}^{(k)}$ to lie on the same loop via a product of two-leg operators - for example $\Gamma^{(1)}\left(x_{1}^{(1)}, \ldots, x_{m_{1}}^{(1)}\right)=Q^{12}\left(x_{1}^{(1)}\right) \ldots Q^{m_{1} 1}\left(x_{m_{1}}^{(1)}\right)$. Each $\Gamma^{(k)}$ uses a distinct set of spin indices, so that $m_{\text {tot }} \equiv \sum_{k=1}^{q} m_{k}$ distinct spin indices are used in total. The same reasoning as for the case $q=1$ gives

$$
\begin{aligned}
\mathcal{L}^{-m_{\text {tot }}}\left\langle\sum_{i_{1}, \ldots, i_{q}}^{\prime} l_{i_{1}}^{m_{1}} \ldots l_{i_{q}}^{m_{q}}\right\rangle & \\
= & f^{m_{\text {tot }}} \frac{n^{q} \Gamma(n) \Gamma\left(m_{1}\right) \ldots \Gamma\left(m_{q}\right)}{\Gamma\left(n+m_{\text {tot }}\right)},
\end{aligned}
$$

again in the thermodynamic limit.

These calculation require integer $m_{\text {tot }} \leq n$. It is useful to relax both the upper limit on $m_{\text {tot }}$ and the restriction to integers. The replica technique achieves the first, simply by evaluating moments for sufficiently large fugacity, then setting $n$ to the required value in final expressions. Supersymmetric $\sigma$ models [22, 23, 25, 27, 28, provide an alternative route to the same conclusions. Separately, allowing non-integer $m$ leads to an interpretation of the coefficient $f$ : since loops with $l_{i} \sim \mathcal{L}$ dominate the lefthand side of Eq. (6) for large $\mathcal{L}$ and $m>1$, by taking the limits $\mathcal{L} \rightarrow \infty$, then $m \rightarrow 1$, we find that $f$ is the fraction of links covered by extended loops. Moreover, by taking a similar limit in Eq. (7), with $q=2$ and $m_{1}, m_{2} \rightarrow 1$, and Eq. (6) at $m=2$, one sees that this fraction has no fluctuations in the thermodynamic limit.

The expressions we have obtained for moments are those of the Poisson Dirichlet distribution, which we now introduce. It may be defined [1] as the limiting case of a Dirichlet distribution for $M$ variables when $M$ diverges. In turn, the Dirichlet distribution is a probability distribution on $M$ variables $y_{i}(i=1,2, \ldots M)$ satisfying $y_{i} \geq 0$ and the constraint $\sum_{i=1}^{M} y_{i}=1$, with the form

$$
\frac{\Gamma(M \alpha)}{[\Gamma(\alpha)]^{M}}\left(y_{1}, y_{2} \ldots y_{M}\right)^{\alpha-1} \mathrm{~d} y_{1} \ldots \mathrm{d} y_{M-1} .
$$

The PD distribution is the limit $M \rightarrow \infty$ and $\alpha \rightarrow 0$ of the Dirichlet distribution with the parameter $\theta=M \alpha$ held fixed, and one obtains from Eq. (8) in this limit

$$
\left\langle\sum_{i_{1}, \ldots, i_{q}}^{\prime} y_{i_{1}}^{m_{1}} \ldots y_{i_{q}}^{m_{q}}\right\rangle=\frac{\theta^{q} \Gamma(\theta) \Gamma\left(m_{1}\right) \ldots \Gamma\left(m_{q}\right)}{\Gamma\left(\theta+m_{\text {tot }}\right)} .
$$


Comparison of Eqns. (7) and (9) shows that: $(i)$ the normalised, ordered lengths $x_{i}=l_{i} /(f \mathcal{L})$ of extended loops are PD distributed, and ( $i i)$ the PD parameter takes the value $\theta=n$ for directed loops with fugacity $n$. These are our central results.

The modifications required to treat undirected loops are straightforward: in this case the $\sigma$ model field $Q$ is parameterised as in Eq. (1) but the vector $\mathbf{z}$ is constrained to be real. Subsequent results hold, but with the replacement of $n$ by $n / 2$. In particular, the PD parameter for undirected loops is $\theta=n / 2$.

To put these results in context it is useful to connect them with what is known about the statistics of loop lengths on shorter scales, by considering in full the distribution $P_{\text {link }}(l)=\mathcal{L}^{-1} l\left\langle\sum_{i} \delta\left(l-l_{i}\right)\right\rangle$ for the length $l$ of a loop passing through a randomly selected link. Consider a system of linear size $L$, so that $\mathcal{L} \sim L^{d}$. The correlation length $\xi$ for the system sets a scale beyond which loops are Brownian [22]. At distances $l \ll \xi$ behaviour is either model-dependent (if $\xi$ is comparable to the lattice spacing) or critical (if $\xi$ is large, a regime we have discussed elsewhere [22]). In the range $\xi \ll l \ll L^{2}$, where loops are insensitive to sample boundaries, their Brownian character implies

$$
P_{\text {link }}(l)=C l^{-d / 2}
$$

with non-universal normalisation $C$. For $L^{2} \ll l \leq f \mathcal{L}$ the distribution crosses over to the PD form

$$
P_{\text {link }}(l)=\theta \mathcal{L}^{-1}(1-l /(f \mathcal{L}))^{\theta-1}
$$

obtained from Eq. (8) by integrating over all but one $y_{i}$. Note that Eqns. (10) and 111 match in order of magnitude at $l \sim L^{2}$, as they should. Note also that in order to see both regimes it is important that the boundary conditions allow only loops, not open strands.

Simulations. As a test and illustration of our results, we next present data from Monte Carlo simulations of three-dimensional loop models described in detail in Ref. [22]. By studying directed loops for integer fugacity $1 \leq n \leq 8$ and undirected loops for $n=1$, we are able to access values of the PD parameter $\theta=1 / 2$ and integer $1 \leq \theta \leq 8$. We use system sizes $40 \leq L \leq 100$ and average over $10^{5}$ loop configurations.

Our results complement earlier simulations of a different model with fixed PD parameter $\theta=1$ [20]. It is interesting to note that there exist other numerical observations of the $\mathrm{PD}$ in literature on loop problems, which have not previously been recognised in these terms: see e.g. Fig. 2 (right panel) of Ref. [5] for $\theta=1 / 2$, Fig 7 of Ref. 7 for $\theta=1$ and Fig. 3 of Ref. 9 for $\theta=2$.

We discuss first results for $P_{\text {link }}(l)$. An overview of the calculated behaviour is given in Fig. 1, where we plot $\mathcal{L} P_{\text {link }}(l)$ versus $l / \mathcal{L}$ for size $L=40$. The two regimes of Eqns. (10) and (11) are evident. Comparison of data from different system sizes (see inset) demonstrates that the loop length for crossover between Brownian and PD forms scales with $L^{2}$. In Fig. 2 we examine the PD regime more closely, showing good agreement between data and theoretical expectations using the fraction $f$ of links on extended loops as the single fitting parameter. The dependence of the distribution on the $\mathrm{PD}$ parameter $\theta$ is striking.

As a further test, independent of any fitting parameter, we compute ratios of moments of the loop length distribution. We define

$$
R \equiv \frac{\left\langle\sum_{i} l_{i}^{4}\right\rangle}{\left\langle\sum_{k} l_{k}^{2}\right\rangle^{2}} \quad \text { and } \quad \mathcal{Q}_{m} \equiv \frac{\left\langle\sum_{i \neq j} l_{i}^{m} l_{j}^{m}\right\rangle}{\left\langle\sum_{k} l_{k}^{m}\right\rangle^{2}} .
$$

From Eq. (7) we expect

$$
R=\frac{6(\theta+1)}{(\theta+3)(\theta+2)}
$$

and

$$
\mathcal{Q}_{m}=\frac{(m-1+\theta)(m-2+\theta) \ldots \theta}{(2 m-1+\theta)(2 m-2+\theta) \ldots(m+\theta)} .
$$

The simulation data shown in Fig. 3 are in excellent agreement with these expressions for $R, Q_{2}, Q_{3}$ and $Q_{4}$.

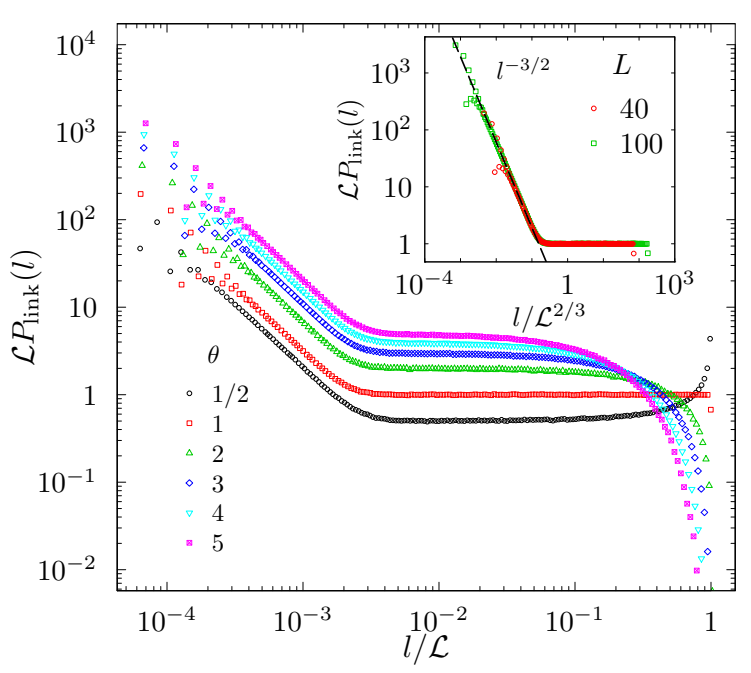

FIG. 1: (Color online) $\mathcal{L} P_{\text {link }}(l)$ vs. $l / \mathcal{L}$, on a double logarithmic scale for the indicated $\theta$ values. Inset: comparison of data for $L=40$ and $L=100$. Dashed line has slope $-3 / 2$. Fluctuations at small $l$ arise from loops of length a few lattice spacings.

Discussion. It is remarkable that the joint probability distribution of lengths of macroscopic loops should have a form that is both calculable and non-trivial. An appealing alternative perspective on this result is provided by arguments that have been developed in the mathematical physics literature [19], which we now summarise.

The starting point is to consider a stochastic process on loop configurations, of a type called split-merge, under which the distribution is stationary. This process is in fact the one that we employ for Monte Carlo simulations 


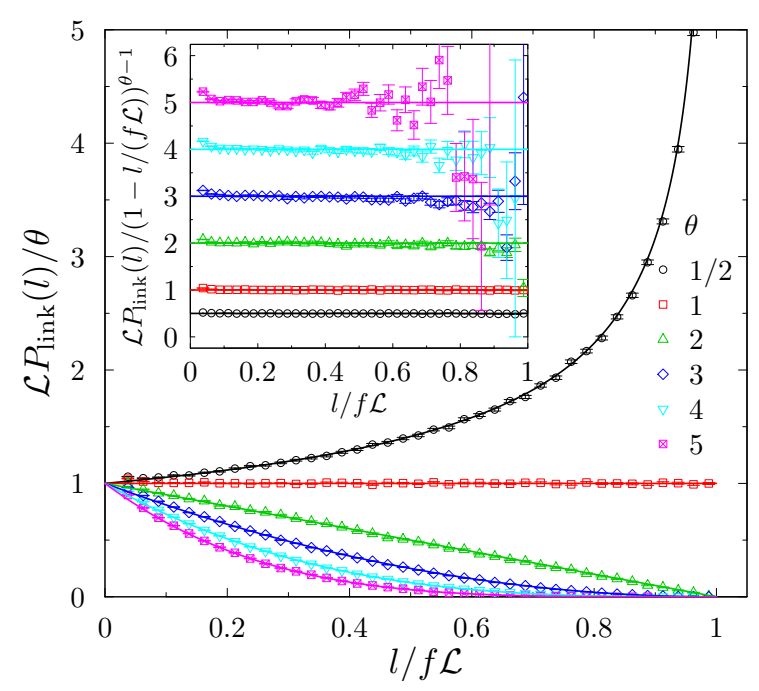

FIG. 2: (Color online) Behaviour of loop length distribution in $\mathrm{PD}$ regime: $\mathcal{L} P_{\text {link }}(l) / \theta$ vs. $l / f \mathcal{L}$, showing dependence on $\mathrm{PD}$ parameter $\theta$. Points: simulation data; curves from Eq. (11). Inset: the measured ratio $\mathcal{L} P_{\text {link }}(l) /(1-l /(f \mathcal{L}))$ is close to its theoretical value $\theta$ except for $l \approx f \mathcal{L}$, where statistical errors are large.

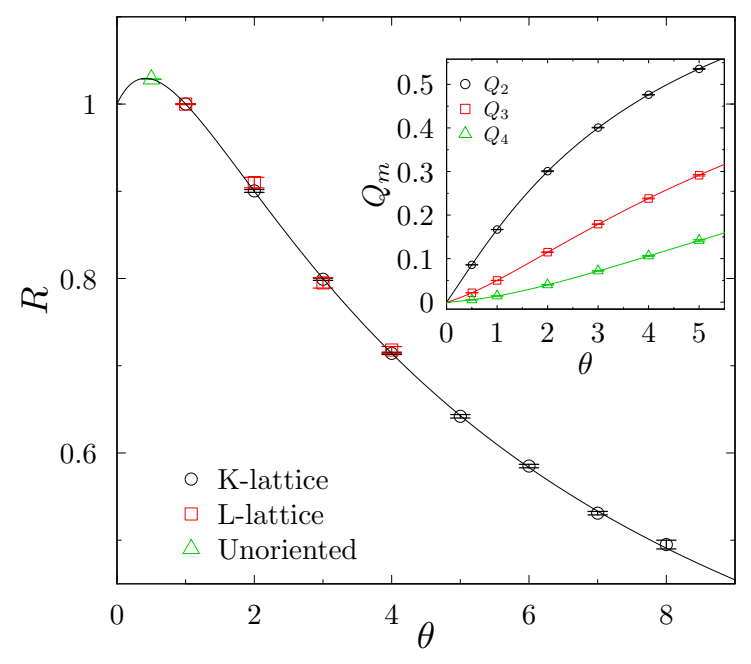

FIG. 3: (Color online) Comparison of simulation data (points) with theoretical values (lines) for ratios of moments of loop lengths. In the main panel we plot the one-loop ratio $R$ and in the inset the two-loop ratios $Q_{2}, Q_{3}$ and $Q_{4}$. Universality is tested by the comparison of results for different lattices (K, $\mathrm{L}$ and Unoriented), defined in Refs. 22, 24] and 25.

[25]. At one time step we pick a node in the lattice at random and compare the colours of the two loop strands that pass through it. These strands are either two parts of a single loop that visits the node twice, or parts of two separate loops that each pass once through the node. If the colours are different we do not change the configuration, but if they are the same, then with a certain probability we break both strands and reconnect the resulting ends in a new pairing. In the case of directed loops, there is only one way to form this new pairing and the effect of the Monte Carlo move is either (if both strands initially belong to a single loop) to split one loop into two, or (if the strands are initially from distinct loops) to merge two loops into one. For undirected loops, since there are two ways to make new pairings, the Monte Carlo move always merges two initial loops into one, but only splits a single loop into two on half of all attempts.

The next stage is to derive consequences for the loop length distribution from the requirement that the rates at which loops split and merge should be equal. Let $\lambda_{1}$ and $\lambda_{2}$ be the lengths of the split loops and $\lambda_{1}+\lambda_{2} \equiv \lambda$ that of the merged loop. To make progress one needs to know the probabilities $\pi_{1}(\lambda)$ for the selected node to lie on one loop, and $\pi_{2}\left(\lambda_{1}, \lambda_{2}\right)$ for it to lie on two. If $\lambda_{1}$ and $\lambda_{2}$ are small, exact statements are impossible since the probabilities encode correlations of the loop ensemble. But when the loops are macroscopic $\left(\lambda_{1,2} \gg L^{2}\right)$ it is plausible to conjecture [19] that they pass through nodes in an uncorrelated way. These probabilities are then simply related to lengths by the expressions

$$
\pi_{1}(\lambda)=\mathcal{L}^{-2} \lambda\left\langle\sum_{i} \delta\left(\lambda-l_{i}\right)\right\rangle \equiv \mathcal{L}^{-1} P_{\text {link }}(\lambda)
$$

and

$$
\pi_{2}\left(\lambda_{1}, \lambda_{2}\right)=\mathcal{L}^{-2} \lambda_{1} \lambda_{2}\left\langle\sum_{i \neq j} \delta\left(\lambda_{1}-l_{i}\right) \delta\left(\lambda_{2}-l_{j}\right)\right\rangle .
$$

For detailed balance we require

$$
\theta \pi_{1}\left(\lambda_{1}+\lambda_{2}\right)=\pi_{2}\left(\lambda_{1}, \lambda_{2}\right),
$$

with $\theta=n$ for directed loops (since merging occurs only if both loops are the same colour) and $\theta=n / 2$ for undirected loops (when, in addition, the rate for splitting is halved). Evaluating the right sides of Eqns. (15) and (16) using the PD yields $\pi_{1}(\lambda)=\mathcal{L}^{-2} \theta[1-\lambda /(f \overline{\mathcal{L}})]^{(\theta-1)}$ and $\pi_{2}\left(\lambda_{1}, \lambda_{2}\right)=\mathcal{L}^{-2} \theta^{2}\left[1-\left(\lambda_{1}+\lambda_{2}\right) /(f \mathcal{L})\right]^{(\theta-1)}$. Hence the $\mathrm{PD}$ is stationary under this split-merge process, provided long loops are sufficiently independent that the conjectured forms for $\pi_{1}(\lambda)$ and $\pi_{2}\left(\lambda_{1}, \lambda_{2}\right)$ are correct.

In summary, we have used the $\sigma$ model formulation of loop problems and replica methods to establish a relation between the Poisson Dirichlet distribution of loop lengths and averages over the spaces $C P^{n-1}$ and $R P^{n-1}$ or $S^{n-1}$.

We thank Y. Fyodorov for a useful discussion. This work was supported in part by EPSRC Grant No. EP/I032487/1 and by the MINECO and FEDER Grants No. FIS2012-38206 and AP2009-0668. 
[1] J. F. C. Kingman, J. Royal Stat. Soc. B 37, 1 (1975).

[2] P. G. de Gennes, Scaling Concepts in Polymer Physics (Cornell University Press, Ithaca, 1979).

[3] J. Oitmass, C. Hamer, and W. Zheng, Series Expansion Methods for Strongly Interacting Lattice Models (Cambridge University Press, Cambridge 2010).

[4] R. J. Baxter, Exactly Solved Models in Statistical Mechanics (Academic Press, London, 1982).

[5] L. D. C. Jaubert, M. Haque and R. Moessner, Phys. Rev. Lett. 107, 177202 (2011).

[6] L. D. C. Jaubert, S. Piatecki, M. Haque, and R. Moessner, Phys. Rev. B 85, 054425 (2012).

[7] V. Khemani, R. Moessner, S. A. Parameswaran, and S. L. Sondhi, Phys. Rev. B 86, 054411 (2012).

[8] S. Liang, B. Doucot, and P. W. Anderson, Phys. Rev. Lett. 61, 365 (1988).

[9] A. F. Albuquerque, F. Alet, and R. Moessner, Phys. Rev. Lett. 109, 147204 (2012).

[10] T. Vachaspati and A. Vilenkin, Phys. Rev. D 30, 2036 (1984).

[11] P. de Gennes and J. Prost, The Physics of Liquid Crystals (Clarendon Press, Oxford, 1993).

[12] K. O'Holleran, M. R. Dennis, F. Flossmann, and M. J. Padgett, Phys. Rev. Lett. 100, 053902 (2008).

[13] R. M. Bradley, J-M. Debierre, and P. N. Strenski, Phys. Rev. Lett 682332 (1992); R. M. Bradley, P. N. Strenski, and J-M. Debierre, Phys. Rev. A 45, 8513 (1992).

[14] B. Derrida, Physica D 107, 186 (1997).

[15] R. P. Feynman, Phys. Rev. 91, 1291 (1953).

[16] For reviews, see: H. G. Evertz, Adv. Phys. 52, 1 (2003);
A. W. Sandvik, AIP Conf. Proc. 1297, 135 (2010); R. K. Kaul, R. G. Melko, and A. W. Sandvik, arXiv:1204.5405.

[17] M. Aizenman and B. Nachtergaele, Comm. Math. Phys., 164, 17 (1994).

[18] O. Schramm, Israel J. Math. 147, 221 (2005).

[19] For a review, see: C. Goldschmidt, D. Ueltschi, and P. Windridge, Contemp. Math. 552, 177 (2011).

[20] S. Grosskinsky, A. A. Lovisolo, and D. Ueltschi, J. Statist. Phys. 146, 1105-1121 (2012).

[21] M. Ortuño, A. M. Somoza, and J. T. Chalker, Phys. Rev. Lett. 102, 070603 (2009).

[22] A. Nahum, J. T. Chalker, P. Serna, M. Ortuño and A. M. Somoza, Phys. Rev. Lett. 107, 110601 (2011).

[23] A. Nahum and J. T. Chalker, Phys. Rev. E 85, 031141 (2012).

[24] A. Nahum, P. Serna, A. M. Somoza and M. Ortuño, Phys. Rev. B. 87, 184204 (2013).

[25] A. Nahum, J. T. Chalker, P. Serna, M. Ortuño and A. M. Somoza, arXiv:1308.0144

[26] We note that a similar idea for $m=2$ has been used in Ref. [29] to relate the two-point correlation function of quantum spin models to their loop representations. The extension to general $m$ necessitates the replica or supersymmetry methods that we introduce here.

[27] N. Read and H. Saleur, Nucl.Phys. B 613, 409 (2001).

[28] C. Candu, J. L. Jacobsen, N. Read, and H. Saleur, J. Phys. A 43, 142001 (2010).

[29] D. Ueltschi, arXiv:1211:4141 (2012). 